\title{
Automatic Thermal Leakage Detection in Building Facades Using Laser and Thermal Images
}

\author{
D. Fernández-Llorca, A.G. Lorente, C. Fernández, \\ I.G. Daza, and M.A. Sotelo \\ Computer Engineering Department, University of Alcalá \\ Alcalá de Henares, Madrid, Spain \\ llorca@aut.uah.es
}

\begin{abstract}
This paper describes an automatic system that detects thermal insulation properties of the different components of buildings envelope by combining laser data with thermal images. Sensor data is obtained from a moving vehicle equipped with a GPS sensor. Range data is integrated to obtain the 3D structure of the building facade, and combined with thermal images to separate components such as walls, windows frames and glasses. Thermal leakage is detected by detecting irregularities in the thermal measurements of each building component separately (window glasses, window frames and walls).
\end{abstract}

Keywords: Thermal imaging, laser, thermal leakage, 3D reconstruction, building envelope.

\section{Introduction}

The use of infrared thermography as a tool to quantitatively identify irregularities in the heat, cold and moisture properties of the buildings envelope has become an important research field in the last few years. Only in the EU the building sector accounts for $40 \%$ of the energy requirements and offers the largest single potential for energy efficiency 11. Thermographic inspections have been usually carried out by thermographers by means of manual labelling of thermal images. Recently, mobile thermal scanning systems have been proposed to capture data from the ground, surveying hundreds of buildings in a short period of time [2. However, the recorded images are off-line analysed including a considerable amount of manual supervision work. Automatic interpretation of thermal images to detect thermal leakage on buildings has been recently proposed, including the detection of building components such as windows and doors 3 .

In this paper, laser data and thermal images are obtained from a moving vehicle equipped with a low-cost GPS sensor. Laser measurements are integrated to obtain the 3D structure of the building facade, and combined with thermal images to separate components such as walls and windows (frames and glasses). Thermal leakage is detected by measuring irregularities in the thermal measurements of each component separately. 


\section{System Overview}

A commercial vehicle has been equipped with an infrared camera, a four layers laser scanner and a GPS system (see Fig. 1), including CAN Bus communication. An overview of the whole system is shown in Fig. 2. An on-line calibration procedure is firstly applied to get both the infrared camera intrinsic and the laserto-camera extrinsic parameters. Laser measurements are integrated to obtain the 3D structure of the building facade and a Finite State Machine (FSM) is then applied to detect the building limits. Range-data is combined with thermal images to separate components such as windows frames, windows glasses and walls. Thermal leakage is finally computed by measuring irregularities in the thermal images of each building component separately.

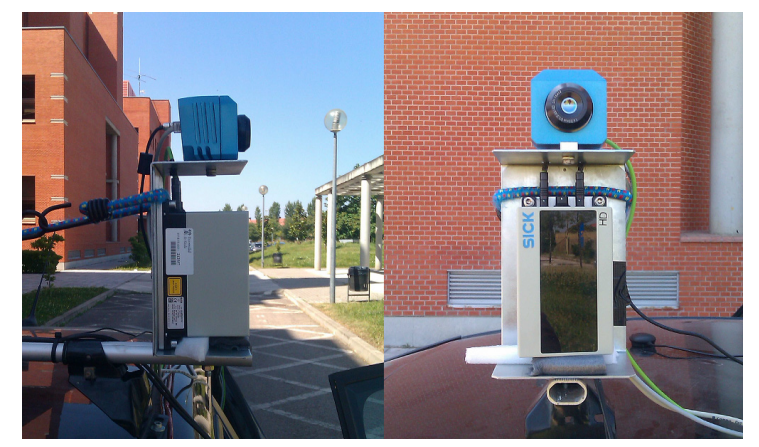

Fig. 1. Laser and infrared camera prototype on-board of the vehicle

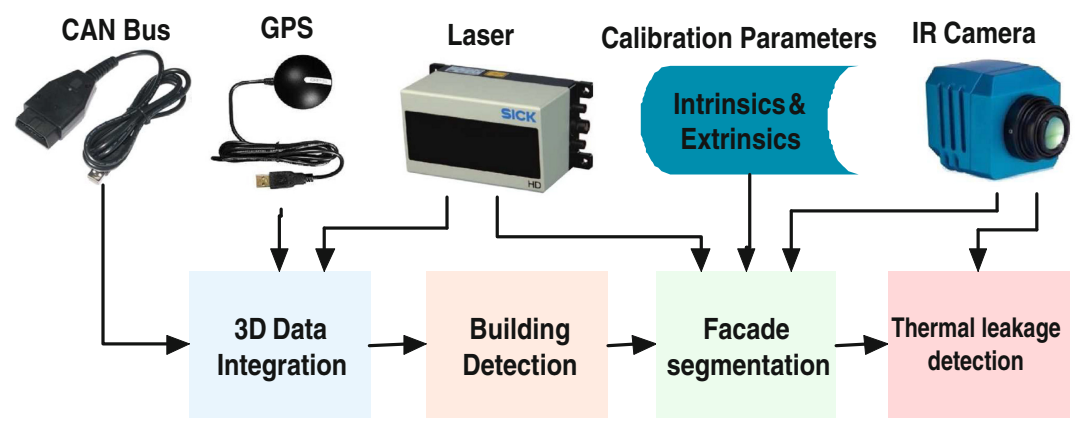

Fig. 2. System overview 


\section{System Calibration}

Intrinsic parameters of the infrared camera are computed by means of a standard calibration process with a chessboard calibration pattern. However, in our case, the chessboard pattern was previously heated up to make visible the squares to the infrared sensor. The extrinsic relationship between the camera and the laser was obtained using the calibration procedure proposed in 4. The calibration process needs extrinsic plane to camera parameters of different poses of the pattern, as well as the laser points on the chessboard, which are only a portion of the whole laser measurements (see Fig. 3). Then, a nonlinear minimization process on the Euclidean distances from laser points to the chessboard planes is accomplished to obtain camera-to-plane rotation matrix and translation vector.

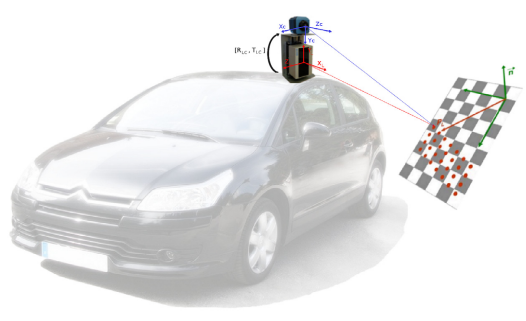

(a)
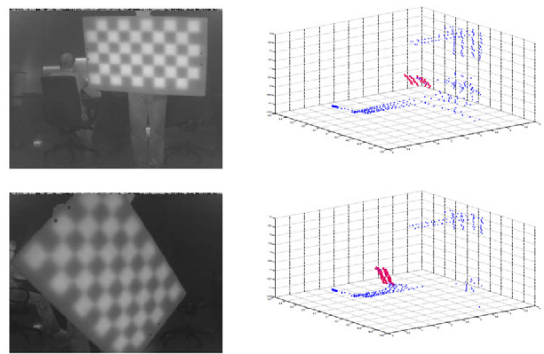

(b)

Fig. 3. (a) Extrinsic camera-laser calibration process. (b) Two examples of infrared images and the corresponding 3D data (red points are labelled as pertaining to the pattern).

\section{3D Data Integration}

As depicted in Fig 1, the laser is vertically oriented. For each infrared image, only four 3D vertical planes are available. In order to have depth information for most pixels of the infrared image, the 3D measurements are transformed from different vehicle positions using the Northing-Easting coordinates supplied by the GPS. The dynamic model is simplified assuming constant elevation between measurements. This constraint does not drastically affect the accuracy of the reconstruction and provides a straightforward solution. Lets consider the scheme showed in Fig. 4. By knowing the global position at intervals $[t-1, t]$ and $[t+$ $N-1, t+N]$ we can compute the following vector and orientations:

$$
\begin{aligned}
\bar{V}_{t-1}^{t} & =\left[\left(E_{t}-E_{t-1}\right),\left(N_{t}-N_{t-1}\right)\right]=\left[E_{t-1}^{t}, N_{t-1}^{t}\right] \\
\bar{V}_{t+N-1}^{t+N} & =\left[\left(E_{t+N}-E_{t+N-1}\right),\left(N_{t+N}-N_{t+N-1}\right)\right]=\left[E_{t+N-1}^{t+N}, N_{t+N-1}^{t+N}\right]
\end{aligned}
$$




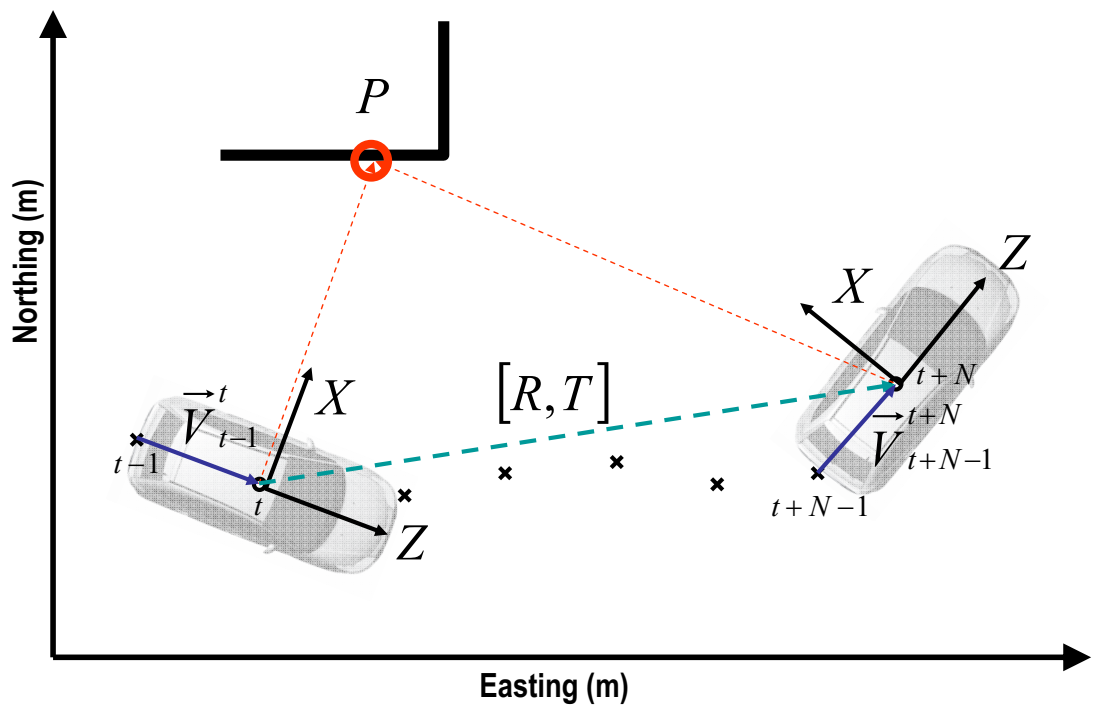

Fig. 4. Graphical scheme of the main components involved when transforming 3D points from one location at time $t$ to a different location at time $t+N$

$$
\theta_{t}=\operatorname{atan}\left(\frac{N_{t-1}^{t}}{E_{t-1}^{t}}\right) ; \theta_{t+N}=\operatorname{atan}\left(\frac{N_{t+N-1}^{t+N}}{E_{t+N-1}^{t+N}}\right)
$$

Both the rotation matrix and the translation vector from $t$ to $t+N$ with respect to $t$ are given by:

$$
\begin{aligned}
& \bar{T}_{t}^{t+N}=\left(\begin{array}{c}
T z_{t}^{t+N} \\
T x_{t}^{t+N}
\end{array}\right)=\left(\begin{array}{cc}
\cos \left(\theta_{t}\right) & \sin \left(\theta_{t}\right) \\
-\sin \left(\theta_{t}\right) & \cos \left(\theta_{t}\right)
\end{array}\right)\left(\begin{array}{c}
E_{t+N}-E_{t} \\
N_{t+N}-N_{t}
\end{array}\right) \\
& \theta_{t}^{t+N}=\theta_{t+N}-\theta_{t} \rightarrow R_{t}^{t+N}=\left(\begin{array}{ccc}
\cos \left(\theta_{t}^{t+N}\right) & \sin \left(\theta_{t}^{t+N}\right) & 0 \\
-\sin \left(\theta_{t}^{t+N}\right) & \cos \left(\theta_{t}^{t+N}\right) & 0 \\
0 & 0 & 1
\end{array}\right)
\end{aligned}
$$

Then, any $3 \mathrm{D}$ point $P$ detected by the laser w.r.t. its position at $t\left(Z_{t}, X_{t}, Y_{t}\right)$, can be transformed w.r.t. its position at $t+N\left(Z_{t}^{t+N}, X_{t}^{t+N}, Y_{t}^{t+N}\right)$ by means of the following equation:

$$
\left(\begin{array}{c}
Z_{t}^{t+N} \\
X_{t}^{t+N} \\
Y_{t}^{t+N}
\end{array}\right)=\left(\begin{array}{ccc}
\cos \left(\theta_{t}^{t+N}\right) & \sin \left(\theta_{t}^{t+N}\right) & 0 \\
-\sin \left(\theta_{t}^{t+N}\right) & \cos \left(\theta_{t}^{t+N}\right) & 0 \\
0 & 0 & 1
\end{array}\right)\left[\left(\begin{array}{c}
Z_{t} \\
X_{t} \\
Y_{t}
\end{array}\right)-\left(\begin{array}{c}
T z_{t}^{t+N} \\
T x_{t}^{t+N} \\
0
\end{array}\right)\right]
$$

Thus, we can obtain $3 \mathrm{D}$ range measurements for one specific image using range data from previous and posterior vehicle locations. A temporal window is used as a function of the vehicle speed (via CAN Bus connection). It is worth to 
mention that the IR camera captures frames at 30Hz. However, GPS signal is received at $10 \mathrm{~Hz}$. In order to compute inner global positions, a linear interpolation between two consecutive GPS measurements is applied using the vehicle speed provided by the CAN Bus $(50 \mathrm{~Hz})$. Fig. 5 depicts one example of the proposed $3 \mathrm{D}$ reconstruction approach.
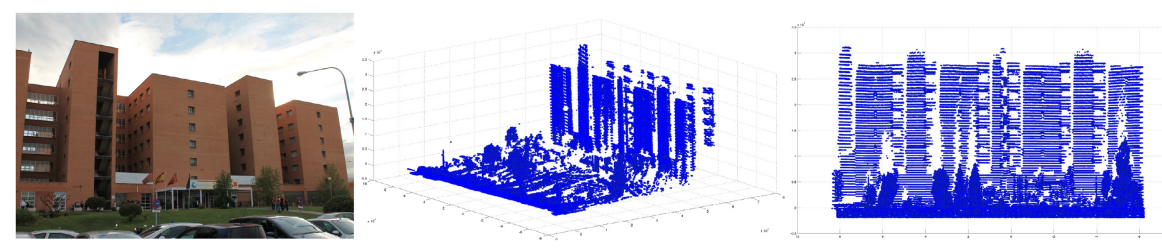

Fig. 5. 3D reconstruction example

\section{Range-Based Building Detection}

To isolate each building as a separated object to be processed in further stages, we use a Finite State Machine (FSM), as depicted in Fig. 6. Four states have been defined: Q0 (no vertical plane), Q1 (init building), Q2 (building) and Q3 (end building). Transitions between states are achieved from frame to frame as a function of the number of iterations during which a vertical plane has been detected $\left(V_{Y E S}\right)$ or not $\left(V_{N O}\right)$. Vertical planes are detected using the laser measurements by means of a Hough transform with a constrained accumulation matrix [5] that only allows lines with vertical orientation. If a vertical plane has been detected in a specific frame, the counter $V_{Y E S}$ is increased. If no vertical plane is detected, the counter $V_{N O}$ is increased. An example of the building detection process can be seen in Fig. 7 .

\section{Facade Segmentation}

The building detection stage provides an image corresponding to the building facade, including thermal information and range measurements. The facade segmentation stage uses this information to provide three main components: windows glasses, windows frames and walls. The proposed procedure is defined as a sequential three-staged process. First, glass material is detected by finding holes in the range image since glass does not reflect the laser beams. Second, windows frames are computed by detecting rectangular contours around the glass regions previously obtained. Finally, the walls are segmented applying a region growing technique in the remaining regions (i.e., without windows frames and glass) of both the infrared image and the range image, whose results are combined using a binary AND operator. The overall facade segmentation procedure with intermediate results can be seen in Fig. 8 . 


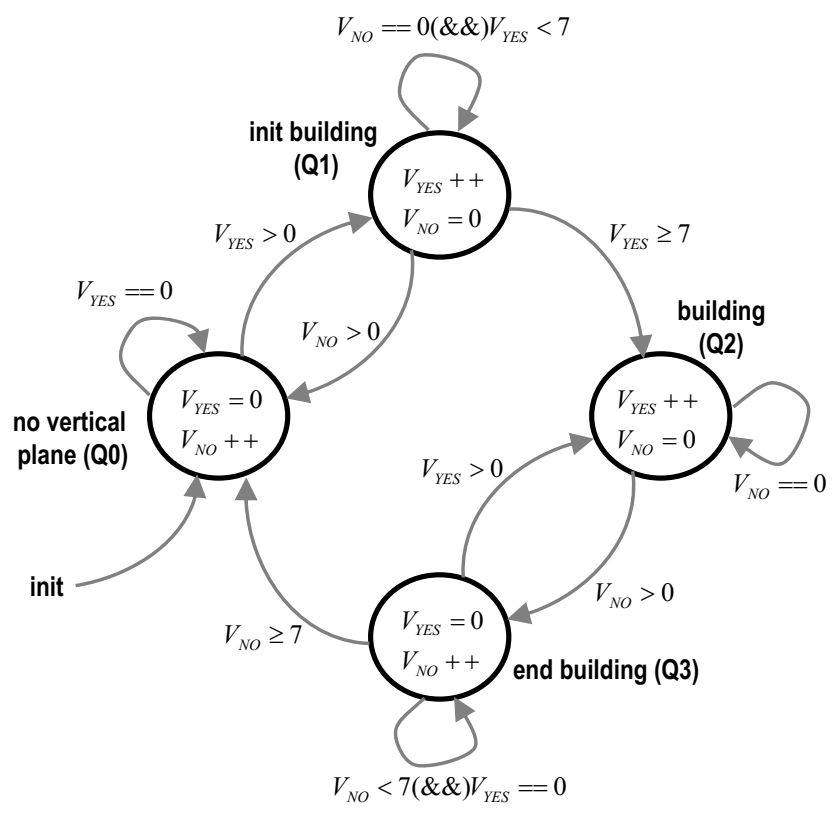

Fig. 6. FSM used for building detection

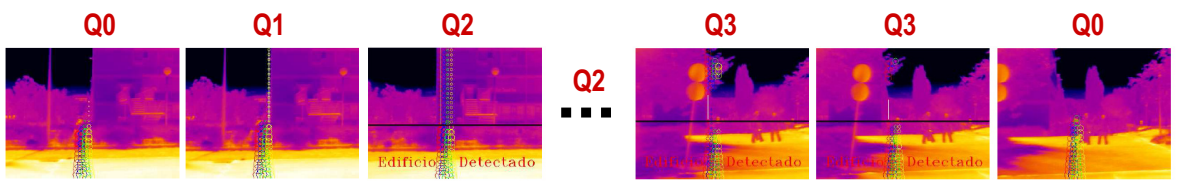

Fig. 7. Range-based building detection example

\section{Thermal Leakage and Irregularities}

After segmenting each one of the building components, thermal leakage and irregularities are detected using the following procedure. For each component, we find local minimum or maximum points (depending on the season of the year) using these points as the seeds of a region growing algorithm. Thus, in summertime local minimum points involve cold leakage, i.e., air conditioner inside the building is being emitted outside. On the other hand, in wintertime local maximum points mean heat leakage, i.e., heat inside the building is being emitted outside. Local minimum/maximum points are required to be $K \sigma^{2}$ times far from the average region value, as given by Eqs. (7) and (8) for summertime and wintertime respectively.

$$
p(u, v) \in R_{i}=\left\{\begin{array}{l}
\text { Seed if }\left|p(u, v)-\bar{m}_{i}\right| \leq K \sigma_{i} \\
\text { No seed otherwise }
\end{array}\right.
$$




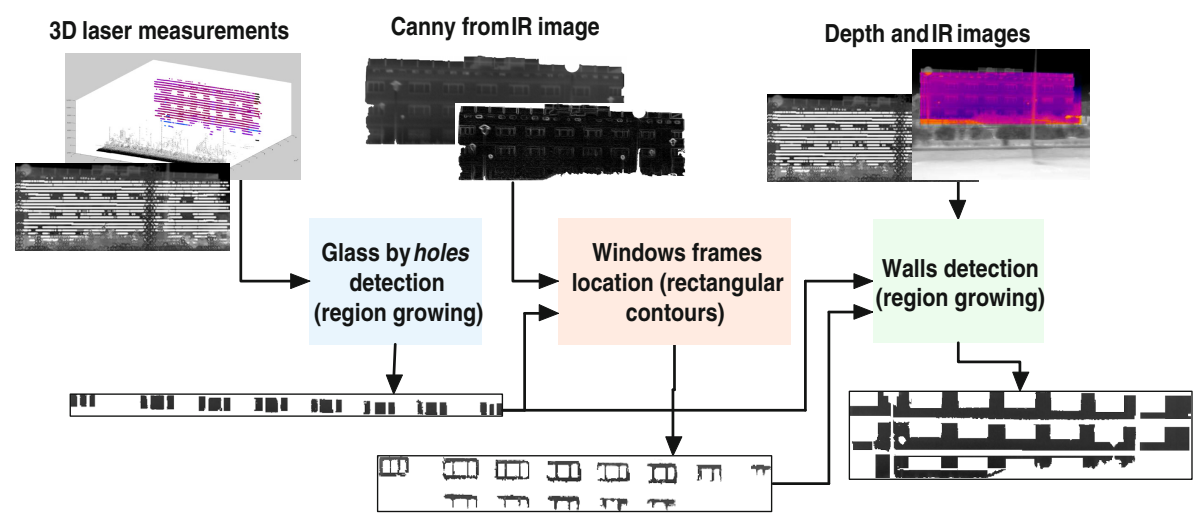

Fig. 8. Facade segmentacion process: overview and partial results

$$
p(u, v) \in R_{i}=\left\{\begin{array}{l}
\text { Seed if }\left|p(u, v)-\bar{m}_{i}\right| \geq K \sigma_{i} \\
\text { No seed otherwise }
\end{array}\right.
$$

where $R_{i}$ is the specific region corresponding to window glasses, window frames or walls, $\bar{m}_{i}$ is the mean value of region $R_{i}$ and $\sigma_{i}$ is the standard deviation corresponding to region $R_{i}$. The value for $K$ is experimentally obtained. Region growing is continually applied until no more seeds are available. The results given by this approach are finally considered as the thermal leakage that will be provided as the global system output.

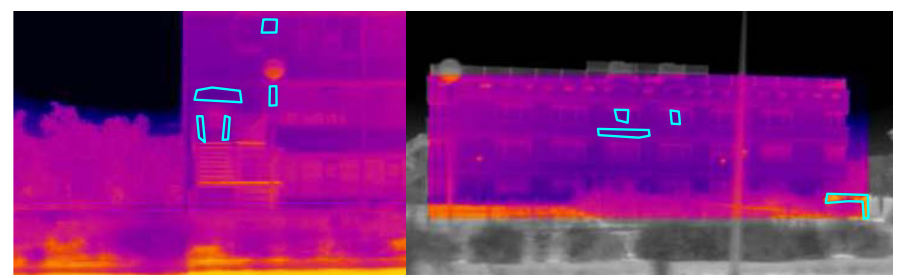

Fig. 9. Thermal irregularities detection in summertime (air conditioner inside the building is emitted outside)

\section{Conclusions and Future Work}

This paper described an automatic thermal leakage detection system. The main contributions w.r.t. previous works are twofold: the combination of laser-based measurements with thermal images, and the thermal irregularities detection for three main components of the building facades (window glasses, window frames and walls). A commercial vehicle was equipped with an IR camera, a four layers 
laser scanner and a GPS system. The extrinsic relationship between the camera and the laser was obtained using an off-line calibration procedure. Range measurements were integrated in the space using the global position of the vehicle. A simple state machine is used to detect the boundaries of the buildings. Each facade is thus isolated, including thermal and range information. 3D data is firstly used to segment windows glasses, since they produce holes in the 3D reconstruction of the facade. Then, rectangular regions surrounding the windows glasses are detected to separate the windows frames. A region growing approach is applied to obtain the remaining areas corresponding to walls. Thermal irregularities are then detected by means of region growing technique that used local minimum/maximum points as seeds.

Future works are mainly focused in obtaining massive results from hundreds of buildings in both summertime and wintertime. The proposed approach will be tested against a manually labelled ground truth. Since the system captures the GPS position of the vehicle and the relative distance between the buildings and the vehicle, thermal analysis will be finally provided in geo-referenced digital maps.

Acknowledgment. This work was supported by the Spanish Ministry of Economy and Competitiveness under Research Grant ONDA-FP TRA2011-27712C02-02.

\section{References}

1. FLIR. Infrared Guidebook for Building Applications. An Informative Guide for the Use of Infrared in the Building Industry. FLIR Systems AB (2009)

2. HeatSeekers (UK), http://www.homeheatseekers.co.uk/about/

3. Sirmacek, B., Hoegner, L., Stilla, U.: An automatic system to detect thermal leakages and damages on building facade using thermal images. In: JURSE (2011)

4. Fernández, C., Gavilán, M., Llorca, D.F., Parra, I., Quintero, R., Lorente, A.G., Vlacic, L.J., Sotelo, M.A.: Free Space and Speed Humps Detection using Lidar and Vision for Urban Autonomous Navigation. In: IEEE Intelligent Vehicle Symposium (2012)

5. García-Garrido, M.A., Ocaña, M., Llorca, D.F., Arroyo, E., Pozuelo, J., Gavilán, M.: Complete Vision-Based Traffic Sign Recognition Supported by I2V Communication System. Sensors 12, 1148-1169 (2012) 